\title{
Random Determinants
}

\author{
R. Fortet
}

\begin{abstract}
Determinants whose elements involve random variables are discussed and expressions derived for the first and second moments. Applications are made to $n$-dimensional geometry, especially, to finding limiting probabilities for the event: "a given point lies above a random hyperplane", under fairly general hypotheses. The random variable $A_{\mathrm{n}} h-B$ is considered, where $A_{n}$ and $B$ are certain minors of the determinantal equation of the random hyperplane, and $h$ is a coordinate of the given point.

An asymptotic expression for $E\left\{A_{n}^{2}\right\}$ is obtained, and it is shown that $E\left\{B^{2}\right\}$ is of the order of $(1 / n) E\left\{A_{n}^{2}\right\}$.
\end{abstract}

1.

In this section real random variables $X_{\imath j}$ depending on two indices $i$ and $j$ and having the following properties are considered:

Let $A_{1}, A_{2}, \ldots, A_{k}$ be $k$ random variables chosen from the $X_{i j}$ 's. Then we make the assumptions that

(a) for any $i$ and $j$, for any $k$, and for any choice of the $A_{l}$ 's, the conditional mathematical expectation

$$
E\left(X_{i j} \mid A_{1}, \ldots, A_{k}\right)=0,
$$

if $X_{i j}$ is not one of the $A_{i}$ 's.

(b) For any $i, j$, for any $k$, and for any choice of the $A_{i}$ 's, the conditional second order moment

$$
E\left(X_{i j}^{2} \mid A_{1}, \cdots, A_{k}\right)=\sigma_{i}^{2},
$$

(where $\sigma_{i}$ is a constant that depends only on $i$ ) provided $X_{i j}$ is not one of the $A_{i}$ 's.

Consider the following random determinant:

We have the

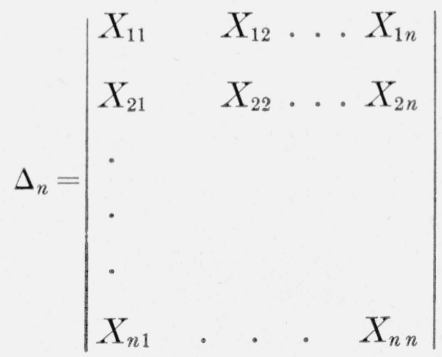

Lemma: $E\left(\Delta_{n}\right)=0$ and $E\left(\Delta_{n}^{2}\right)=n ! \prod_{i=1}^{n} \sigma_{i}^{2}$.

Proof. Let $A_{i j}$ be the cofactor of $X_{i j}$ in $\Delta_{n}$; we can write

$$
\begin{gathered}
\Delta_{n}=\sum_{j} A_{1 j} X_{1 j} \\
E\left(\Delta_{n}\right)=\sum_{j} E\left\{A_{1 j} E\left(X_{1 j} \mid A_{1 j}\right)\right\} .
\end{gathered}
$$

From (a), $E\left(X_{1 j} \mid A_{1 j}\right)=0$; hence

${ }_{1}$ The preparation of this paper was sponsored (in part) by the Office of the Air Comptroller, USAF.
Also

$$
E\left(\Delta_{n}\right)=0 \text {. }
$$

Now

$$
\Delta_{n}^{2}=\sum_{j} A_{1 j}^{2} X_{1 j}^{2}+\sum_{j \neq k} A_{1 j} A_{1 k} X_{1 j} X_{1 k} .
$$

$$
\begin{aligned}
& E\left\{\sum_{j \neq k} A_{1 j} A_{1 k} X_{1 j} X_{1 k}\right\}= \\
& \sum_{j \neq k} E\left\{A_{1 j} A_{1 k} X_{1 j} E\left(X_{1 k} \mid A_{1 j}, A_{1 k}, X_{1 j}\right)\right\}
\end{aligned}
$$

From (a) $E\left(X_{1 k} \mid A_{1 j}, A_{1 k}, X_{1 j}\right)=0$; hence

$$
E\left\{\Delta_{n}^{2}\right\}=\sum_{j} E\left\{A_{1 j}^{2} X_{1 j}^{2}\right\}=\sum_{i} E\left\{A_{1 j}^{2} E\left(X_{1 j}^{2} \mid A_{1 j}\right)\right\},
$$

and from (b) this reduces to

$$
E\left\{\Delta_{n}^{2}\right\}=\sigma_{1}^{2} \sum_{j} E\left(A_{1 j}^{2}\right) .
$$

Now $A_{1 j}$ is a $\Delta_{n-1}$, which depends only on those $X_{i j}$ with $i \neq 1$. If we assume that the formula

$$
E\left\{\Delta_{n}^{2}\right\}=n ! \sum_{i=1}^{n} \sigma_{i}^{2}
$$

is true for $n=1,2, \ldots, r$, (1) shows that (2) is also valid for $n=r+1$; on the other hand (2) obviously holds for $n=1$. Thus (2) is established by induction, and the proof of the lemma is complete.

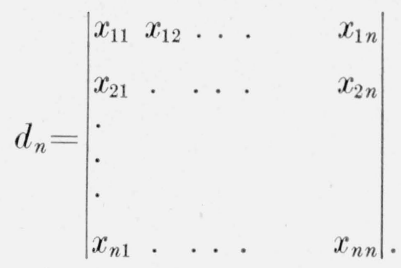

Nonrandom real numbers $x_{1 j}$ and the determinant are now considered.

Suppressing the $k$ rows $i_{1}, i_{2}, \ldots, i_{k}$ in $d_{n}$, a rectangular matrix is obtained with $(n-k)$ rows; $T\left[i_{1}, i_{2}, \ldots, i_{k} ; d_{n}\right]$ is called the sum of the squares of all determinants of $(n-k)$ th order deduced from this matrix. 
Let $D_{n}$ be the following random determinant:

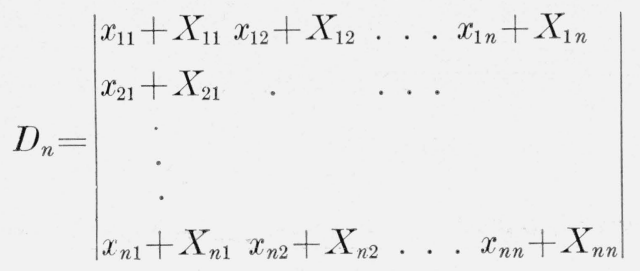

$D_{n}$ can be written as a sum of elementary determinants, the elements of which are either $x_{i j}$ or $X_{i j}$ but never $x_{i j}+X_{i j}$. One of these elementary determinants is $d_{n}$; all the other elementary determinants are random determinants. Let $S$ be one of these random determinants: $S$ consists of $k$ rows of $X_{i j}$ 's $(k \geq 1)$ and $(n-k)$ rows of $x_{i j}$ 's. For instance, suppose that the first row of $S$ consists of the $X_{1 j} ; S$ is a linear homogeneous form in the $X_{1 j}$ 's, the coefficients of which are independent of the $X_{1 j}$ 's. Thus according to (a) $E(S)=0$; consequently $E\left(D_{n}\right)=d_{n}$.

Let $i_{1}, i_{2}, \ldots, i_{k}$ be the $k$ rows of $S$ consisting of some $X_{i j}$ 's, that is, row $i_{1}$ consists of the $X_{i_{1} j}$, row $i_{2}$ of the $X_{i_{2} j}$, etc. Employing the Laplace development of $S$ in terms of these $k$ rows $i_{1}, i_{2}$, $\ldots, i_{k}$, we can write $S$ in the form:

$$
S=\Sigma^{*} \Delta_{k}\left(j_{1}, j_{2}, \ldots, j_{k}\right) B\left(j_{1}, j_{2}, \ldots, j_{k}\right),
$$

where $\Delta_{k}\left(j_{1}, j_{2}, \ldots, j_{k}\right)$ is a determinant of the preceding $\Delta$ type, the elements of which are those $X_{i j}$ with $i \in i_{1}, i_{2}, \ldots, i_{k}, j \in j_{1}, \ldots, j_{k}$; $B\left(j_{1}, j_{2}, \ldots, j_{k}\right)$ is the algebraic complement of $\Delta_{k}\left(j_{1}, j_{2}, \ldots, j_{k}\right)$; and the summation $\Sigma^{*}$ is extended over all combinations $\left(j_{1}, j_{2}, \ldots, j_{k}\right)$ of order $k$ taken from the $n$ integers $1,2, \ldots, n$. For two different combinations we have:

$$
E\left\{\Delta_{k}\left(j_{1}, j_{2}, \ldots, j_{k}\right) \cdot \Delta_{k}\left(j_{1}^{\prime}, \ldots, j_{k}^{\prime}\right)\right\}=0,
$$

since there is at least one $j_{\alpha}, j_{1}$ say, which is not a $j_{\beta}^{\prime}$, so that the product $\Delta_{k}\left(\dot{j}_{1}, \ldots, j_{k}\right) \cdot \Delta_{k}\left(j_{1}^{\prime}, \ldots\right.$ $\left.j_{k}^{\prime}\right)$ is a homogeneous linear form in $X_{i_{1} j_{1}}, X_{i_{2} j_{1}}$, ... $X_{i_{k} j_{1}}$, and (3) follows from (a). Consequently

$$
E\left(S^{2}\right)=\Sigma^{*} B^{2}\left(j_{1}, \ldots, j_{k}\right) E\left\{\Delta_{k}^{2}\left(j_{1}, \ldots, j_{k}\right)\right\},
$$

and by the lemma,

$$
\begin{aligned}
E\left(S^{2}\right) & =\sum^{*}\left(B^{2}\left(j_{1}, \ldots, j_{k}\right) \cdot k ! \prod_{\alpha=1}^{k} \sigma_{i_{\alpha}}^{2}\right) \\
& =k ! \prod_{\alpha=1}^{k} \sigma_{i_{\alpha}}^{2}\left[\sum^{*} B^{2}\left(j_{1}, \ldots, j_{k}\right)\right] .
\end{aligned}
$$

But obviously,

$$
\Sigma * B^{2}\left(j_{1}, \ldots, j_{k}\right)=T\left(i_{1}, i_{2}, \ldots, i_{k} ; d_{n}\right) .
$$

Hence we have

$$
E\left(S^{2}\right)=T\left(i_{1}, i_{2}, \ldots, i_{k} ; d_{n}\right) k ! \prod_{\alpha=1}^{k} \sigma_{i_{\alpha}}^{2} .
$$

Consider the product of two different elementary determinants $S_{1}$ and $S_{2}$. There is at least one row consisting of some $X_{i j}$ that appear in $S_{1}$ and not in $S_{2}$, or in $S_{2}$ and not in $S_{1}$. For example, suppose the first row in $S_{1}$, consisting of the $X_{1 j}$ 's, does not appear in $S_{2}$; then the product $S_{1} S_{2}$ is a linear homogeneous form in the $X_{1 j}$ 's, the coefficients of which are independent of the $X_{1 j}$ 's. Consequently by (a), $E\left(S_{1} S_{2}\right)=0$ and $E\left(D_{n}^{2}\right)$ reduces to the sum of the squares of all the elementary determinants. This gives us the following:

Theorem 1: Under assumptions (a) and (b),

$$
\begin{aligned}
& E\left(D_{n}\right)=d_{n} \\
& E\left(D_{n}^{2}\right)=\sum_{k=0}^{n} k !\left(\sum^{*} T\left(i_{1}, i_{2}, \ldots, i_{k} ; d_{n}\right) \prod_{\alpha=1}^{k} \sigma_{i_{\alpha}}^{2}\right) .
\end{aligned}
$$

In (5) the summation $\Sigma^{*}$ is extended over all combinations $\left(i_{1}, i_{2}, \ldots, i_{k}\right)$ of order $k$ of the integers 1 , $2, \ldots, n$; for convenience we put

$$
T\left(1,2, \ldots, n ; d_{n}\right)=1 .
$$

An interesting feature of formulas (4) and (5) is that these formulas do not depend on the probability laws of the $X_{i j}$ 's. This fact remains valid even if the $\sigma_{i}$ 's depend on $j$, but (5) becomes more complicated; on the other hand it is possible to compute the higher moments of $D_{n}$ by using similar reasoning and suitable assumptions. However, the formulas seem quite complicated. It also appears difficult to obtain the probability law of $D_{n}$, even under such hypotheses as that the $X_{i j}$ are normally distributed.

Of course if $\sigma_{1}=\sigma_{2}=\ldots=\sigma_{n}=\sigma$, where $\sigma$ is a constant independent of $i,(5)$ reduces to:

$$
E\left(D_{n}^{2}\right)=\sum_{k=0}^{n} T_{k}\left(d_{n}\right) k ! \sigma^{2 k},
$$

where

$$
T_{k}\left(d_{n}\right)=\Sigma^{*} T\left(i_{1}, \ldots, i_{k} ; d_{n}\right) .
$$

From (5) certain interesting results may be deduced, which are perhaps already known. These results are connected with the geometrical interpretation of the co efficients of (5). Let $E_{n}$ be an $n$-dimensional Euclidean space, with orthogonal coordinates. The $n$ numbers $x_{i 1}, x_{i 2}, \ldots, x_{i n}$ may be regarded as the coordinates of a point $P_{i}$ in $E_{n}$, and $x_{i 1}+X_{i 1}, x_{i 2}+X_{i 2}, \ldots$, $x_{i n}+X_{i n}$ can be considered as the coordinates of a random point $M_{n}$ in $E_{n}$. That $d_{n}$ is invariant under orthogonal transformations is well known. If $\left(0, P_{1} P_{2} \ldots P_{n}\right)$ is the volume in $E_{n}$ of the parallelepiped formed by the vectors $\overrightarrow{0 P}_{1}, \overrightarrow{0 P}_{2}, \ldots$, $\overrightarrow{0 P}_{n}$ we have:

$$
d_{n}= \pm\left(0, P_{1} P_{2} \ldots P_{n}\right) .
$$

An analogous interpretation holds for $D_{n}$ and $\left(0, M_{1} M_{2} \ldots M_{n}\right)$; hence $E\left(D_{n}^{2}\right)$ is invariant under an orthogonal transformation. If the $X_{i j}$ 's are normally distributed, this property also persists. The 
$\sigma_{i}$ remain unaltered also. We conclude that the coefficients $T\left(i_{1}, \ldots, i_{k} ; d_{n}\right)$ are invariant.

$T\left(i_{1}, \ldots, i_{k} ; d_{n}\right)$ depends only on $P_{i_{1}}, \ldots, P_{i_{k}}$; consider the set of points $P_{i_{1}}^{\prime}, P_{i_{2}}^{\prime}, \ldots, P_{i_{k}}^{\prime}$ defined as follows:

$$
\begin{aligned}
& {\overrightarrow{0 P_{i_{1}}^{\prime}}}^{\prime}=\overrightarrow{0 P}_{i_{1}} \\
& {\overrightarrow{0 i_{i_{2}}}}^{\prime}={\overrightarrow{0 P_{i_{2}}}}_{\lambda_{21}} \overrightarrow{0 P}_{i_{1}}
\end{aligned}
$$

$$
{\overrightarrow{0 P_{i}}}^{\prime}=\overrightarrow{0 P}_{i_{k}}+\lambda_{k 1} \overrightarrow{0 P}_{i_{1}}+\ldots+\lambda_{k, k-1} \overrightarrow{0 P}_{i_{k-1}}
$$

where the $\lambda_{i j}$ are such that the vectors ${\overrightarrow{0 P_{i}}}_{i_{1}}, \overrightarrow{0 P}_{i_{2}}^{\prime}$, $\ldots, \overrightarrow{0 P_{i_{k}}^{\prime}}$ are mutually orthogonal. From a classical property of determinants we can replace $P_{i_{1}}, \ldots$, $P_{i_{k}}$ by $P_{i_{1}}^{\prime}, \ldots, P_{i_{k}}^{\prime}$ without altering $T\left(i_{1}, \ldots, i_{k} ; d_{n}\right)$. The invariance of $T\left(i_{1}, \ldots, i_{k} ; d_{n}\right)$ under orthogonai transformations implies that the vectors $\overrightarrow{0 P}_{1_{\alpha}}^{\prime}(\alpha=1$, $\ldots, k)$ can be taken as coordinate axes. Then it becomes obvious that

$$
T\left(i_{1}, \ldots, i_{k} ; d_{n}\right)=\left(0, P_{i_{1}}^{\prime} \ldots P_{i_{k}}^{\prime}\right)^{2},
$$

the volume $\left(0, P_{i_{1}}^{\prime} \ldots P_{i_{k}}^{\prime}\right)$ being considered as a volume in a $k$-dimensional subspace of $E_{n}$; but it is also obvious that

$$
\left(0, P_{i_{1}}^{\prime} \ldots P_{i_{k}}^{\prime}\right)^{2}=\left(0, P_{i_{1}} P_{i_{2}} \ldots P_{i_{k}}\right)^{2},
$$

and we have established:

Theorem 2:

$$
T\left(i_{1}, \ldots, i_{k} ; d_{n}\right)=\left(0, P_{i_{1}} \ldots P_{i_{k}}\right)^{2},
$$

and consequently,

$$
T_{k}\left(d_{n}\right)=\sum^{*}\left(0, P_{i_{1}} \ldots P_{i_{k}}\right)^{2} .
$$

On the other hand, considering $P_{1}, \ldots, P_{n}$ as fixed points in $E_{n}$, and $\Omega$ as a moving point, $\left(\Omega, P_{i_{1}} \ldots\right.$. $\left.P_{i_{k}}\right)^{2}$ and $\Sigma^{*}\left(\Omega, P_{i_{1}} \ldots P_{i_{k}}\right)^{2}$ are given by $(6)$ or (7) under a simple change of the origin of coordinates. If one puts

and

$$
\left(\Omega, P_{i_{1}} \ldots P_{i_{k}}\right)^{2}=C
$$

$$
\Sigma^{*}\left(\Omega, P_{i_{1}} \ldots P_{i_{k}}\right)^{2}=C^{*},
$$

where $C$ and $C^{*}$ are any positive constants, then eq (8) and (9) define quadrics $Q\left(i_{1}, \ldots, i_{k} ; C\right)$ and $Q_{k}\left(C^{*}\right)$, respectively, of ellipsoidal type. Thus it follows from (5) that the relation

$$
E\left\{\left(\Omega, M_{1} \ldots M_{n}\right)^{2}\right\}=e,
$$

where $e$ is some positive constant, also defines a quadric ? (e) (which is in general an ellipsoid).

\section{2 .}

In Euclidean $n$-space with the coordinates defined as above consider $n$ fixed points $P_{1}, P_{2}, \ldots, P_{n}$, and a fixed point $S$ on the axis $0 x_{n}$ with coordinates $(0,0, \ldots ., 0, h)$. Let $M_{1}, M_{2}, \ldots, M_{n}$ be $n$ random points, and consider the random plane $\Pi$, (i. e., an $(n-1)$-dimensional linear variety) defined by $\left(M_{1}\right.$, $\left.M_{2}, \ldots, M_{n}\right)$. The equation of $I I$ has the following determinantal form

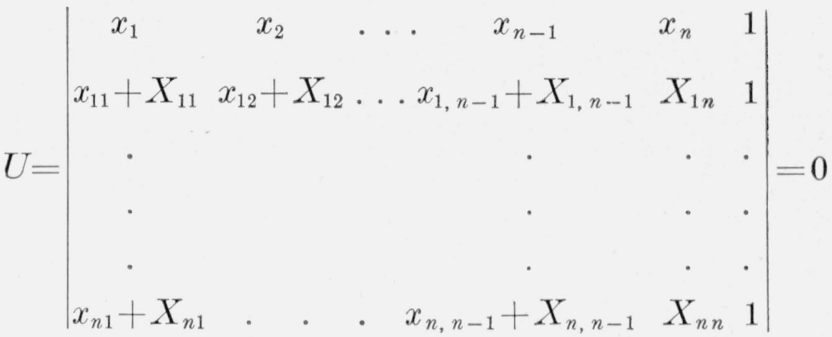

Developing this determinant in terms of the elements of the first row and their cofactors, one obtains

$$
U=\sum_{j=1}^{n} A_{j} x_{j}-B=0 .
$$

For convenience we set

$$
Y_{i j}=x_{i j}+X_{i j} \quad(i=1,2, . ., n ; j=1,2, . ., n-1) .
$$

Let $M_{i}^{\prime}$ be the point $\left(Y_{i 2}, \ldots Y_{i, n-1}, 0\right)$ and $V$ the linear variety of $n-2$ dimensions defined by

$$
x_{n}=0, \quad U=0,
$$

and let $H_{i}$ be the orthogonal projection of $M_{i}$ or $M_{i}^{\prime}$ on $V$. An elementary computation shows that, in absolute value,

$$
\frac{\overline{M_{i}^{\prime} M_{i}}}{\overline{H_{i} M_{i}^{\prime}}}=\frac{\left|X_{i n}\right|}{\overline{H_{i} M_{i}^{\prime}}}=\frac{\sqrt{\sum_{j=1}^{n-1} A_{j}^{2}}}{\left|A_{n}\right|}
$$

We now suppose that:

(A) the distance between $P_{i}$ and $\mathrm{P}_{j}$ is equal to 1 , for any $i$ and $j$. In this case the $n$ points $P_{i}$ form a regular polyhedron in the variety $x_{n}=0$; this polyhedron admits $\left(\begin{array}{l}n \\ k\end{array}\right)$ linear varieties $V_{i j}$ as varieties of symmetry, $V_{i j}$ being defined as that $(n-2)$-dimensional variety formed by the points in $x_{n}=0$ which are equidistant from $P_{i}$ and $P_{j}(i \neq j)$. Now suppose that $V$ is a moving $(n-2)$-dimensional variety, and consider the quantity

$$
s=\max _{i}\left(\text { distance from } V \text { to } P_{i}\right) .
$$


$s$ attains its minimum value when $V$ is a $V_{i j}$, in which case $s=\frac{1}{2}$. Consequently in all cases there is at least one point $P_{i}$ such that the distance from $V$ to $P_{i}$ is at least $\frac{1}{2}$.

Now suppose that $n$ tends to $+\infty$ with condition (A) always holding; we make the following assumptions:

(B) $\lim _{n \rightarrow \infty} \operatorname{Pr}\left\{\operatorname{Max}\left|X_{i n}\right|<\epsilon\right\}=1 \quad$ for any $\epsilon>0$;

(C) there exists a positive number $a<\frac{1}{2}$ and independent of $n$ such that:

$$
\lim _{n \rightarrow \infty} \operatorname{Pr}\left\{\operatorname{Max}_{i} \sum_{j=1}^{n} X_{i j}^{2}<a^{2}\right\}=1 .
$$

If for instance the distance from $P_{i}$ to $V$ is a maximum when $i=1$, then except in cases of small probability:

$$
\overline{H_{1} M_{1}^{\prime}}>\frac{1}{2}-a,
$$

and

Thus we have:

$$
\left|X_{1 n}\right|<\epsilon .
$$

Theorem 3: Under hypotheses (A), (B), and (C),

$$
\lim _{n \rightarrow \infty} \operatorname{Pr}\left\{\frac{\sqrt{\sum_{1}^{n-1} A_{j}^{2}}}{\left|A_{n}\right|}>0\right\}=0 .
$$

Remark: Assumptions (B) and (C) are not particularly restrictive. They are satisfied, for instance if the $X_{i j}$ 's are independent and normally distributed with

$$
E\left(X_{i j}^{2}\right)=\frac{\rho^{2}}{n},
$$

$\rho$ being $<\frac{1}{2}$ and independent of $i, j, n$; also when the $M_{i}$ are independently distributed with uniform probability density over the interior of the sphere of center $P_{i}$ and radius $\rho<\frac{1}{2}$.

Denote the center of gravity of $P_{1}, \ldots, P_{n}$ by $G$, with coordinates $\left(\xi_{1}, \ldots, \xi_{n}\right)$ given by

$$
\xi_{j}=\frac{1}{n} \sum_{i=1}^{n} x_{i j}, \quad(j=1,2, \ldots, n),
$$

$\xi_{n}=0$. Let $K$ be the center of gravity of $M_{1}$, . . ., $M_{n}$ with coordinates $\xi_{j}+Z_{j}(j=1,2, . ., n)$. Put

$$
Z_{j}=\frac{1}{n} \sum_{i=1}^{n} X_{i j}
$$

We make the following assumptions:

(D) the distance $\overline{O G}$ remains bounded as $n \rightarrow+\infty$;
(E) the $X_{i j}$ satisfy assumptions (a) and (b) of section 1, with

$$
\sigma_{i} \leq \frac{\rho}{\sqrt{n}}
$$

where $\rho^{2}$ is a constant independent of $i$ and $n$. The algebraic distance $L$ from $G$ to $I$ is equal to:

$$
L=\frac{\sum_{j=1}^{n-1} A_{j} \xi_{j}-B}{\sqrt{\sum_{j=1}^{n} A_{j}^{2}}} .
$$

$K$ belongs to $\Pi$, so that

$$
|L| \leq \overline{G K} .
$$

But the coordinates of the vector $\overrightarrow{G K}$ are the $Z_{j}$ 's defined by (16). From (E) it then follows that

$$
E\left(\overline{G K}^{2}\right) \leq \frac{\rho^{2}}{n} .
$$

Hence $\overline{G K}$ and a fortiori $L$ tend toward zero in probability. Write (17) as

$$
\begin{aligned}
\frac{\sum_{j=1}^{n-1} A_{j} \xi_{j}-B}{\sqrt{\sum_{j=1}^{n} A_{j}^{2}}}= & -\frac{B}{A_{n}} \cdot \frac{A_{n}}{\left|A_{n}\right| \sqrt{1+\frac{\sum_{1}^{n-1} A_{j}^{2}}{\left|A_{n}\right|^{2}}}} \\
& +\frac{\sum_{j=1}^{n-1} A_{j} \xi_{j}}{\sqrt{\sum_{n}^{n-1} A_{i}^{2}}} .
\end{aligned}
$$

We have by the Schwartz inequality

$$
\left|\sum_{j=1}^{n-1} A_{j} \xi_{j}\right| \leq \sqrt{\sum_{j=1}^{n-1} A_{j}^{2}} \cdot \sqrt{\sum_{j=1}^{n-1} \xi_{i}^{2}}
$$

so that

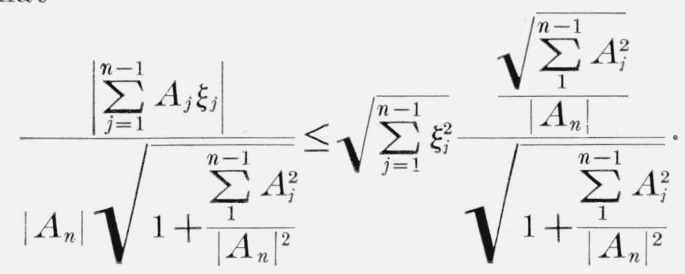

Now $\sqrt{\sum_{j=1}^{n-1} \xi_{i}^{2}}$ is bounded and $\sqrt{\sum_{1}^{n-1} A_{i}^{2}} /\left|A_{n}\right|$ tends to zero in probability. Therefore under the assumptions $(\mathrm{A})-(\mathrm{E})$ we have:

Lemma: As $n \rightarrow \infty,\left|B / A_{n}\right|$ tends to zero in probability.

The algebraic distance from $S$ to $\Pi$ is 


$$
\begin{aligned}
\Delta & =\frac{h A_{n}-B}{\sqrt{\sum_{j=1}^{n} A_{j}^{2}}}=\frac{h A_{n}-B}{\left|A_{n}\right| \sqrt{1+\frac{\sum_{1}^{n-1} A_{j}^{2}}{\left|A_{n}\right|^{2}}}} \\
& =\left(h \frac{A_{n}}{\left|A_{n}\right|}-\frac{B}{\left|A_{n}\right|}\right) \frac{1}{\sqrt{1+\frac{\sum_{1}^{n-1} A_{j}^{2}}{\left|A_{n}\right|^{2}}}} \cdot
\end{aligned}
$$

Suppose that $h>0$; then $S$ is above II if $\Delta A_{n}>0$, or what is the same thing, $\left(h A_{n}^{2}-B A_{n}\right)>0$. We may write this last expression as

$$
h-\frac{B}{A_{n}}>0 .
$$

Thus according to the above lemma (and under the same hypotheses, viz., assumption (A)-(E)):

Theorem 4. If $h>0$, the probability that $S$ is above II tends to 1 when $n \rightarrow+\infty$; if $h<0$, this probability tends to zero.

The case $h=0$ is not clear-cut. But if, for instance, the $M_{i}$ 's are independent with the same continuous probability law which is symmetric about the plane $x_{n}=0$, then clearly $\operatorname{Pr}\{S>\pi\}=\frac{1}{2}$.

\section{3.}

Keeping the notations of section 2, we assume that $\overline{P_{i} P_{j}}=1$ for any $i, j$ and that the $X_{i j}$ 's satisfy conditions (a) and (b) of section 1 with $\sigma_{1}=\ldots=\sigma_{n}=$ $\rho / \sqrt{n}$ where $\rho$ is a constant independent of $i$ and $n$. The other assumptions, (B), (C), and (D) of section 2 , will not be required. We wish to obtain some information about the random variable:

$$
H=A_{n} h-B \text {. }
$$

'Theorem 1 implies immediately that

$$
E(H)=V_{n} h,
$$

where $V_{n}$ is the determinant

$$
V_{n}=\left|\begin{array}{ccc}
x_{11} & x_{12} \ldots x_{1, n-1} & 1 \\
x_{21} & x_{22} \ldots x_{2, n-1} & 1 \\
\cdot & \cdot & \cdot \\
\cdot & \cdot & \cdot \\
\cdot & x_{n 2} \ldots x_{n, n-1} & 1
\end{array}\right| .
$$

$V_{n}$, as we have seen, is given by

$$
V_{n}= \pm\left(P_{1}, P_{2} P_{3} \ldots P_{n}\right) .
$$

That is to say (cf. formula (33) of the Appendix)

$$
V_{n}= \pm \sqrt{\frac{n}{2^{n-1}}}
$$

Let $w_{n}$ be the determinant

$$
\left|\begin{array}{ccc}
x_{11} & x_{12} \ldots x_{1, n-1} & 0 \\
x_{21} & \ldots x_{2, n-1} & 0 \\
\cdot & \cdot & \cdot \\
\cdot & \cdot & \cdot \\
\cdot & \ldots x_{n, n-1} & 0
\end{array}\right| .
$$

For $E\left(H^{2}\right)$ we have at once:

$$
E\left(H^{2}\right)=h^{2} E\left(A_{n}^{2}\right)+E\left(B^{2}\right) .
$$

Since $B$ is a determinant of the form $D_{n}$ (section 1 ) formula $(5 a)$ gives the result that

$$
E\left(B^{2}\right)=\sum_{k=1}^{n} T_{k}\left(w_{n}\right) k ! \frac{\rho^{2 k}}{n^{k}} .
$$

$A_{n}$ is not exactly of type $D_{n}$, but in entirely similar fashion we find

$$
E\left(A_{n}^{2}\right)=\sum_{k=0}^{n-1} T_{k}^{\prime}\left(V_{n}\right) k ! \frac{\rho^{2 k}}{n^{k}} .
$$

$T_{k}^{\prime}\left(V_{n}\right)$ is the sum of the minors of order $(n-k)$ of $V_{n}$ obtained without suppressing the last column of $V_{n}$. For instance

$$
T_{k}^{\prime}\left(i_{1}, \ldots, i_{k} ; V_{n}\right)=\left(P_{j_{1}}, P_{j_{2}} P_{j_{3}} \ldots P_{j_{n-k}}\right)^{2},
$$

where $j_{1}, j_{2}, \ldots, j_{n-k}$ run through all the numbers $1,2, \ldots, n$ except $i_{1}, i_{2}, \ldots, i_{n-k}$. Hence

$$
T_{k}^{\prime}\left(i_{1}, \ldots, i_{k} ; V_{n}\right)=V_{n-k}^{2}=\frac{n-k}{2^{n-k-2}}
$$

and

$$
T_{k}^{\prime}\left(V_{n}\right)=\left(\begin{array}{l}
n \\
k
\end{array}\right)(n-k) 2^{-n+k+1} .
$$

Substituting this expression into (24) one finds

$$
\begin{aligned}
E\left(A_{n}^{2}\right) & =\sum_{k=0}^{n-1}\left(\begin{array}{l}
n \\
k
\end{array}\right)(n-k) 2^{-n+k+1} \cdot k ! \frac{\rho^{2 k}}{n^{k}} \\
& =\frac{n}{2^{n-1}} \sum_{k=0}^{n-1} \frac{(n-1) \ldots(n-k)}{n^{k}}\left(2 \rho^{2}\right)^{k} .
\end{aligned}
$$

If we suppose that $2 \rho^{2}<1,(27)$ yields immediately:

$$
E\left(A_{n}^{2}\right) \sim \frac{1}{1-2 \rho^{2}} \cdot \frac{n}{2^{n-1}},
$$

as $n \rightarrow \infty$.

$B$ is of the form $D_{n}$; we know that $Q_{k}\left(C^{*}\right)$ is a quadric, and it must have as planes of symmetry the $\left(\begin{array}{l}n \\ 2\end{array}\right)$ planes of symmetry $V_{i j}$ of the polyhedron 
$\left(P_{1}, P_{2}, \ldots, P_{n}\right)$. Consequently the center of $Q_{k}\left(C^{*}\right)$ is $G$, and $Q_{k}\left(C^{*}\right)$ is a sphere since $\left(\begin{array}{l}n \\ 2\end{array}\right)>n$, and we can write:

$$
T_{k}\left(w_{n}\right)=\lambda_{k}(n) \overline{O G}^{2}+T_{k}^{*}\left(w_{n}\right),
$$

where $T_{k}^{*}\left(w_{n}\right)$ is the value of $T_{k}\left(w_{n}\right)$ when $\overrightarrow{O G}=0$. On the other hand

$$
T_{k}\left(i_{1}, \ldots, i_{k} ; w_{n}\right)=\left(O, P_{j_{1}} P_{j_{2}} \ldots P_{j_{n-k}}\right),
$$

where $j_{1}, j_{2}, \ldots, j_{n-k}$ are all the numbers $1,2, \ldots$, $n$ except $i_{1}, i_{2}, \ldots, i_{k}$. If $O=G$, we find (cf. appendix, formula $(36))$ :

and

$$
T^{*}\left(i_{1}, \ldots, i_{j} ; w_{n}\right)=\frac{k}{n 2^{n-k}}
$$

Hence

$$
T_{k}^{*}\left(w_{n}\right)=\left(\begin{array}{l}
n \\
k
\end{array}\right) \frac{k}{n 2^{n-k}} .
$$

$\sum_{k=1}^{n} T_{k}^{*}\left(w_{n}\right) k ! \frac{\rho^{2 k}}{n^{k}}=\frac{1}{n 2^{n}} \sum_{k=1}^{n} k \frac{n(n-1) \ldots(n-k+1)}{n^{k}}\left(2 \rho^{2}\right)^{k}$.

If $2 \rho^{2}<1$, (29) becomes asymptotically

$$
\sum_{k=1}^{n} T_{k}^{*}\left(w_{n}\right) k ! \frac{\rho^{2 k}}{n^{k}} \sim \frac{1}{n 2^{n}} \cdot \frac{1 \rho^{2}}{\left(1-2 \rho^{2}\right)^{2}} .
$$

We can now compute the value $T_{k}^{* *}\left(w_{n}\right)$ of $T_{k}\left(w_{n}\right)$ for $P_{n}=O$ (cf. appendix, formula (38)). Writing

$$
T_{k}^{* *}\left(w_{n}\right)=\lambda_{k}(n) \overline{G P}_{n}^{2}+T_{k}^{*}\left(w_{n}\right),
$$

we have an equation which determines $\lambda_{k}(n)$; we find

$$
\lambda_{k}(n)=\frac{n(n-2)(n-3) \ldots(n-k)}{(k-1) ! 2^{n-k-1}}
$$

and

$\sum_{k=1}^{n} \lambda_{k}(n) k ! \frac{\rho^{2 k}}{n^{k}}=\frac{1}{2^{n-1}} \sum_{k=1}^{n} \frac{n(n-2)(n-2) \ldots(n-k)}{n^{k}} k\left(2 \rho^{2}\right)^{k}$.

Asymptotically this expression becomes, for $2 \rho^{2}<1$,

$$
\sum_{k=1}^{n} \lambda_{k}(n) k ! \frac{\rho^{2 k}}{n^{k}} \sim \frac{1}{2^{n-1}} \frac{2 \rho^{2}}{\left(1-2 \rho^{2}\right)^{2}} .
$$

We verify that $E\left(B^{2}\right)$ is of the order of $(1 / n) E\left(A_{n}^{2}\right)$, which agrees with the lemma of section 2 . In the particular case when $G=O, E\left(B^{2}\right)$ is of the order of $\left(1 / n^{2}\right) E\left(A_{n}^{2}\right)$.

\section{Appendix}

Given $n$ points $P_{1}, \ldots, P_{n}$ in an $(n-1)$-dimensional Euclidean space $E_{n-1}$ such that $\overline{P_{i} P_{i}}=1$ for any $i$ and $j$ $(i \neq j)$ (that is, these points are the vertices of a regular polyhedron $\boldsymbol{P}_{n}$ ) we wish to find an expression for the volume (1/n!) $\left|V_{n}\right|$ of $\boldsymbol{P}_{n}$ in $E_{n-1}$. Let $G_{i}$ ke the center of gravity of the points $P_{1}, P_{2}, \ldots, P_{i} ; h_{n}$ the distance between $G_{n-1}$ and $P_{n}$; and $d_{n}$ the distance between $G_{n}$ and any $P_{i}$. We have

$$
\begin{gathered}
\left|V_{n}\right|=h_{n}\left|V_{n-1}\right| \\
(n-1) \overrightarrow{G_{n} G_{n-1}}+\overrightarrow{G_{n} P_{n}}=0 .
\end{gathered}
$$

$G_{n-1}, G_{n}, P_{n}$ are on the same "straight line" (one-dimensional linear variety); and $P_{1} G_{n-1} P_{n}$ is a right triangle; hence:

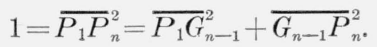

It follows that

$$
\begin{aligned}
d_{n} & =\frac{n-1}{n} h_{n} \\
h_{n}^{2} & =1-\left(\frac{n-2}{n-1}\right)^{2} h_{n-1}^{2} .
\end{aligned}
$$

Clearly $h_{3}$ is known; the recurrence equation (32) can be solved for $h_{n}$ :

$$
h_{n}=\frac{1}{\sqrt{2}} \sqrt{\frac{n}{n-1}} .
$$

It follows by recurrence from (31), $\left|V_{3}\right|$ being equal to $\sqrt{3} / 2$. that:

On the other hand,

$$
\left|V_{n}\right|=\sqrt{\frac{n}{2^{n-1}}}
$$

$$
d_{n}=\frac{1}{\sqrt{2}} \sqrt{\frac{n-1}{n}},
$$

and

$$
{\overline{G_{n} G_{n-1}^{2}}}_{2}^{2} \frac{1}{2(n-1) n}=\frac{1}{2}\left(\frac{n}{n-1}-\frac{1}{n}\right) .
$$

We now compute the volume $\left(G_{n}, P_{1} P_{2} \ldots P_{n-k}\right)$. If $L_{n k}$ is the orthogonal projection of $G_{n}$ on the linear variety $\left(P_{1} P_{2} \ldots P_{n-k}\right)$, we have:

$$
\left(G_{n}, P_{1} P_{2} \ldots P_{n-k}\right)^{2}=\bar{G}_{n} L_{n k}^{2}\left|V_{n-k}\right|^{2} .
$$

But $L_{n k}=G_{n-k}$, so that

$$
{\overline{G_{n} L_{n k}}}_{n}^{2}={\overline{G_{n} G_{n-1}^{2}}}^{2}+{\overline{G_{n-1} G_{n-2}}}_{2}^{2}+\ldots+{\overline{G_{n-k+1} G_{n-k}^{2}}}^{2} .
$$

As in (35),

and so

$$
{\overline{G_{n} L_{n k}}}_{2}^{2}=\frac{1}{2}\left(\frac{1}{n-k}-\frac{1}{n}\right)=\frac{k}{2 n(n-k)},
$$

$$
\left(G_{n}, P_{1} \ldots P_{n-k}\right)^{2}=\frac{k}{n 2^{n-k}} .
$$

We can also compute $\left(P_{n}, P_{1} P_{2} \ldots P_{n-k}\right)$ :

$$
\begin{aligned}
& \left(P_{n}, P_{1} P_{2} \ldots P_{n-k}\right)^{2}={\overline{P_{n} L_{n k}}}^{2}\left|V_{n-k}\right|^{2} \\
\bar{P}_{n} L_{n k}^{2} & ={\overline{P_{n} G_{n-1}}}^{2}+\bar{G}_{n-1} G_{n-2}^{2}+\ldots+{\overline{G_{n-k+1}}}_{n-k}^{2} \\
& =\frac{1}{2}\left(1+\frac{1}{n-k}\right) .
\end{aligned}
$$

Therefore

$$
\left(P_{n}, P_{1} \ldots P_{n-k}\right)^{2}=\frac{(n-k+1)}{2^{n-k}} .
$$

From (37) it follows that

$$
T_{k}^{* *}\left(w_{n}\right)=\left(\begin{array}{l}
n-1 \\
k-1
\end{array}\right) \frac{n-k+1}{2^{n-k}},
$$

Los Angeles, January 11, 1951. 